\title{
Development of a microbiological preparation for crops based on Bacillus pumilus strains
}

\author{
Angelina Malkova*, Ivan Evdokimov, Maxim Shirmanov, Alena Irkitova, and Dina Dudnik \\ EC "Prombiotech", Altai State University, Lenin Avenue 61, 656049, Barnaul, Russian Federation
}

\begin{abstract}
Data of the microbial biopreparation development for protection and crop growth stimulation on the Bacillus bacteria basis are presented. Three B. pumilus strains isolated from the Altai region (the Russian Federation) plants rhizosphere were selected as active components of the bacterial preparation. L-bulone was chosen as the nutrient medium for flasks cultivation of the inoculum. A molasses-based nutrient medium was used to incubate the bacilli in a 15-liter fermenter. The finished microbial preparation was obtained in dry form. The biopreparation is a powder consisting of a lyophilically dried concentrates mixture of genus Bacillus spores. Bacilli biomass were pre-mixed with a protective medium based on gelatin and sucrose. The final number of bacteria in the microbial preparation is $1.29( \pm 0.30) \times 10^{12} \mathrm{CFU} / \mathrm{g}$.
\end{abstract}

\section{Introduction}

The USA, China, Brazil, Argentina and Russia are among the top agricultural producing countries. The protection of plants from various phytopathogens is currently one of the most important problem of this sector of the national economy. The use of plant protection chemicals in agriculture is widespread. It does a negative impact on human and animal health, as well as on the environment in general. Therefore, in recent years there has been an active development of environmentally friendly agriculture through the creation of biological preparations based on microorganisms [1].

Microbial biopreparations are live cells of microorganisms selected for useful properties. They may also contain metabolites [2]. The finished form of biological preparation can be the fluid culture of microorganisms [3]. In this case additional cleaning operations will not be necessary. Microbial cells suspended in various buffers also belong to liquid preparations [4]. This form of products is the most common, despite a number of disadvantages, such as: short shelf life, inconvenient transportation, instability of properties, etc. It is because the creation of biological preparations in this form is the least energy-consuming and labor-intensive, requiring no additional equipment or monetary investment. The preparative form of the microbial product can also be in the form of a paste or thickened cell biomass, gel, etc. [5]. If the target product is produced in dry form, it may be necessary to introduce high-tech processes into the production cycle. Cryopreservation

\footnotetext{
* Corresponding author: gelishka96@mail.ru
} 
or lyophilization can be used to preserve the properties and extend the shelf life of the biopreparation [6].

Molds, yeasts, actinomycetes, and other bacteria are widely used in biotechnological production. Biopreparations containing not a monoculture, but an entire consortium of microorganisms have gained wide popularity in recent years. It is because the microbial composition is more resistant to various environmental factors and has enhanced metabolic capabilities [7].

Bacteria of the genus Bacillus have a high biotechnological potential. Microbial preparations based on these microorganisms are widely used in modern agriculture. "Phytosporin-M", "Alirin-B", and "Bactofit" are the most popular bacillus-based biopreparations for crop production in the Russian Federation. These plant protection products are active against root rot, seed mold, Phytophthora, Alternaria, Rhizoctonia, Oidium, etc. [8]. They all contain the most popular species among bacilli, B. subtilis [9]. At present, scientists are actively studying the effectiveness of other representatives of the genus Bacillus with antifungal and growth-stimulating effects on crops. Researchers in the United States determined that a $B$. pumilus (MSH) strain synthesizes a compound that inhibits the growth of such phytopathogens as Mucor and Aspergillus [10]. Russian scientists have found that a consortium of B. amyloliquefaciens, B. badius, B. gibsonii, $B$. methyloprophicus, B. pumilus, B. simplex, Brevibacterium halotolerans and Pantoea agglomerans strains is more effective in inhibiting plant phytopathogens than each of the strains alone [11]. Therefore, the creation of polycomponent preparations for agriculture based on bacilli is promising.

The aim of this research was to develop a microbiological preparation for crops based on a consortium of three B. pumilus strains.

\section{Materials and Methods}

Three B. pumilus strains from EC "Prombiotech" collection of microorganisms were used in this study (Table 1). The bacteria were isolated from the Altai Krai (the Russian Federation) plants rhizosphere. The strain B. pumilus 16 was deposited in the All-Russian Collection of Industrial Microorganisms (VKPM) with number B-13250 and patented (RF Patent No. 2694522).

Table 1. Objects of the study.

\begin{tabular}{|c|c|c|}
\hline Strain & Source & Collecting ground \\
\hline B. pumilus 4 & Berteroa incana rhizosphere & Altai district, Aya village \\
\hline B. pumilus 7 & Rumex acetos rhizosphere & Altai district, Aya village \\
\hline B. pumilus 16 & Cichorium intybus rhizosphere & $\begin{array}{c}\text { Pavlovsky district, Novye } \\
\text { Zori village }\end{array}$ \\
\hline
\end{tabular}

L-nutrient medium was used to obtain a mother culture and to count the number of bacteria. Bacillus plates were incubated for $18-24 \mathrm{~h}$ at $37^{\circ} \mathrm{C}$ in a thermostat. B. pumilus strains were grown in $500 \mathrm{ml}$ flasks in a shaker incubator under similar conditions with rotation at $250 \mathrm{rpm}$ [12]. The working volume of the fermenter is about 10-12 L, and the mother culture proportion should be about $10 \%$. Therefore, the starter culture of each strain was prepared in $1 \mathrm{~L}$ volume.

B. pumilus strains were cultured in a fermenter on nutrient media of the following composition (\%): 2.5 molasses, 1.25 corn extract, 0.1 yeast extract, 0.05 peptone, 0.025 $\mathrm{MgSO}_{4}, 0.003 \mathrm{MnSO}_{4}, 0.1 \mathrm{CoCl}_{2}$. Saline solution at the rate of $10 \mu 1$ per $1 \mathrm{~L}$ was added to the medium. The salt liquid consisted of $1 \mathrm{~g} \mathrm{FeSO}_{4}, 1 \mathrm{~g} \mathrm{CuSO}_{4}$ and $100 \mathrm{ml}$ of distilled water. 
Initial conditions in the bioreactors: sterile air supply rate $-0.5 \mathrm{~L} / \mathrm{min} ; \mathrm{pH}-6,8-7,0$; pressure $-0,2-0,3 \mathrm{~atm}$.; stirring speed $-250 \mathrm{rpm}$; temperature $-37^{\circ} \mathrm{C}$. The cultivation time was 18-24 hours.

Samples from the fermenter were taken for microscopy and optical density (OD) measurements at $490 \mathrm{~nm}$ to control the biotechnological process.

The bioreactor culture liquid upon completion of the fermentation process was centrifuged at $4100 \mathrm{rpm}$ for 20 minutes. Then the cell biomass was mixed with the protective medium $(2.5 \%$ gelatin and $10 \%$ sucrose $)$ and frozen. The spores were then lyophilized to produce dry bacterial concentrates [13].

\section{Results and Discussion}

The results in table 2 shows that all of studied strains reached a high abundance within the same order of magnitude when cultured in flasks. For all B. pumilus strains OD and $\mathrm{pH}$ were similar and within optimal values. According to bacilli culture microscopic studies, vegetative cells predominate in the microscope field after 18-24 h of cultivation. Spores were not actually detected. All of the above indicates that L-broth is suitable as a nutrient medium for the selected strains inoculum cultivation.

Table 2. Mother culture indices of B. pumilus strains.

\begin{tabular}{|c|c|c|c|}
\hline Strain & $\begin{array}{c}\text { Number, } \\
\text { CFU/ml }\end{array}$ & OD & pH \\
\hline B. pumilus 4 & $3.21( \pm 0.27) \times 10^{9}$ & $0.386 \pm 0.039$ & $7.08 \pm 0.13$ \\
\hline B. pumilus 7 & $1.53( \pm 0.38) \times 10^{9}$ & $0.268 \pm 0.066$ & $6.85 \pm 0.18$ \\
\hline B. pumilus 16 & $2.01( \pm 0.55) \times 10^{9}$ & $0.365 \pm 0.068$ & $6.73 \pm 0.28$ \\
\hline
\end{tabular}

Table 3 shows the dynamics of Bacillus cultures OD in a 15-liter fermenter. B. pumilus 4 changed the medium OD faster, but all strains reached approximately the same value of this index by the end of fermentation. The $\mathrm{pH}$ of $B$. pumilus 4 culture reached the value $7.31 \pm 0.12$, B. pumilus $7-7.13 \pm 0.06$, and B. pumilus $16-8.04 \pm 0.21$ by the end of the fermentation process.

Table 3. OD change by hours of bioreactor cultivation

\begin{tabular}{|c|c|c|c|c|}
\hline \multirow{2}{*}{ Strain } & \multicolumn{4}{|c|}{ OD by hours } \\
\cline { 2 - 5 } & $\mathbf{2}$ & $\mathbf{4}$ & $\mathbf{6}$ & $\mathbf{1 8 - 2 4}$ \\
\hline B. pumilus 4 & $0.648 \pm 0.173$ & $1.401 \pm 0.259$ & $1.818 \pm 0.322$ & $2.290 \pm 0.365$ \\
\hline B. pumilus 7 & $0.592 \pm 0.170$ & $1.172 \pm 0.247$ & $1.584 \pm 0.352$ & $2.284 \pm 0.124$ \\
\hline B. pumilus 16 & $0.573 \pm 0.106$ & $1.083 \pm 0.074$ & $1.589 \pm 0.389$ & $2.382 \pm 0.064$ \\
\hline
\end{tabular}

By the interval of 18-24 $\mathrm{h}$ from the fermentation beginning OD ceases to change, microscopic analysis reveals the spores predominance over vegetative cells, oxygen is no longer consumed. All of it signaled the end of the bacilli cultivation process in the fermenter. The fermentation indicates of the genus Bacillus strains differed slightly, but this was not significantly affect the duration of cultivation.

The cells number of all studied strains after lyophilic drying increased by an order of magnitude compared to the cells number of the fermenter culture liquid (Table 4). The highest CFU/g value from the fermenter was observed for B. pumilus 16 strain. After drying the highest cells number was characteristic of $B$. pumilus 7 concentrate. 
Table 4. Changes in the bacilli number during production processes

\begin{tabular}{|c|c|c|c|}
\hline Strain & $\begin{array}{c}\text { Number in the } \\
\text { fermenter, CFU/ml }\end{array}$ & $\begin{array}{c}\text { Number in the } \\
\text { lyophilized } \\
\text { concentrate, } \text { CFU/g }\end{array}$ & $\begin{array}{c}\text { Number in the } \\
\text { finished } \\
\text { preparation, CFU/g }\end{array}$ \\
\hline B. pumilus 4 & $1.61( \pm 0.59) \times 10^{10}$ & $2.91( \pm 0.79) \times 10^{11}$ & \multirow[t]{3}{*}{$1.29( \pm 0.30) \times 10^{12}$} \\
\hline B. pumilus 7 & $1.36( \pm 0.06) \times 10^{10}$ & $5.77( \pm 0.89) \times 10^{11}$ & \\
\hline B. pumilus 16 & $3.77( \pm 0.33) \times 10^{10}$ & $2.64( \pm 0.33) \times 10^{11}$ & \\
\hline
\end{tabular}

The lyophilized biomass weight of genus Bacillus bacteria was about 70-80 g for each strain. Concentrates of all three bacilli strains were mixed in a $1: 1: 1$ ratio to obtain the finished preparation. The practically established cells number of the biopreparation corresponded to mathematical calculations based on the B. pumilus strains CFU/g addition.

A high number of bacterial cells characterizes the finished microbial preparation. Therefore, it is assumed to have good biological activity. It will allow to get the desired effect using small doses of biopreparation. This fact also shows the economic profitability of the new bacterial preparation for plants.

\section{Conclusion}

1. L-broth is a favorable nutrient medium for the cultivation of tested strains mother culture.

2. The molasses-based medium in the fermenter increases the number of B. pumilus $4, B$. pumilus 7, B pumilus 16 to $10^{10} \mathrm{CFU} / \mathrm{g}$. It could not be done in flasks.

3. Lyophilic drying increases the bacteria number by another order of magnitude to $10^{11}$.

4. The biopreparation obtained by equal proportions mixing of three B. pumilus strains concentrates has a final cells number of $1.29( \pm 0.30) \times 10^{12} \mathrm{CFU} / \mathrm{g}$.

\section{Acknowledgements}

The research was carried out within the framework of the Scientific and Pedagogical Employees Support Program of FSFEI HE "Altai State University". Project "Evaluation of microbial biopreparation effectiveness in control of crops phytopathogens".

\section{References}

1. R.R. Azizbekyan, Appl. Biochem, Microbiol., 55(8), 816 (2019)

2. S.S. Murodova, K.D. Davranov, Biotechnologia Acta, 7(6), 92 (2014)

3. A.P. Kozhemyakov, Yu.V. Laktionov, T.A. Popova, A.G. Orlova,A.L Kokorina, O.B. Vaishlya, E.V. Agafonov, S.A. Guzhvin, A.A. Churakov, M.T. Yakovleva, Agricultural Biology, 50(3), 371 (2015)

4. W. Nopcharoenkul, P. Pinphanichakarn, O. Pinyakong, J. of Appl. Microb., 111(1), 36 (2011)

5. J.A. Salamatova, O.M. Minaeva, E.E. Akimova, Tomsk State University Journal of Biology, 1(9), 20 (2010)

6. L. Bircher, A. Geirnaert, F. Hammes, C. Lacroix, C. Schwab, Microb.Biotechnol., 11(4), 721 (2018)

7. X. Qiana, L. Chena, Yu. Suib, C. Chenb, W. Zhangac, J. Zhouac,W. Dongac, M. Jiangac, F. Xinac, K. Ochsenreitherd, Biotech. Advances, 40, 107500 (2019) 
8. E.M. Prikhodko, S.S. Avdeenko, Conference "Development of scientific, creative and innovative activities of young people, 394 (2018)

9. N. Khan, P. Martínez-Hidalgo, T.A. Ice, M. Maymon, E.A. Humm, N. Nejat, E.R. Sanders, D. Kaplan, A.M. Hirsch, Front. Microbiol., 9, 2363 (2018)

10. E.J. Bottone, J. of Med. Microb., 52(1), 69 (2003)

11. H. Mohamed, A.M. Peterson, G.G. Tkachenko, Izvestiya of Saratov University, 16 (4), 420 (2016)

12. A.N. Irkitova, A.V. Grebenshchikova, Ukr. j. ecol, 8(4), 445 (2018)

13. M.B. Kupletskaya, A.I. Netrusov, Microbiology, 80(6), 851 (2011) 